\title{
Evaluación del efecto de los SNPs G530A (calpaína), C357G (calpastatina), G1795A (miopaladina) y G1181A (PPARGC1A) sobre la terneza de carne bovina de una región del Estado de Veracruz, México
}

\author{
Evaluation of the effect of G530A (calpain), C357G (calpastatin), G1795A (myopaladin) and G1181A \\ (PPARGC1A) SNPs, on beef tenderness of a region of Veracruz State, Mexico
}

\begin{abstract}
Ana Elvia Sánchez Mendozaa, ${ }^{b *}$, Salvador Fonseca Coronadoc, Carlos Ignacio Soto Zárate ${ }^{d}$, Luis Humberto López Hernández ${ }^{e}$, Rosalia Meléndez Pérez', José Francisco Montiel Sosaa.

a Laboratorio de Biotecnología en Alimentos y Análisis de DNA Mitocondrial.

b Posgrado en ciencias de la producción y sanidad animal.

Laboratorio de Inmunobiología de enfermedades infecciosas.

d Laboratorio de Morfología Veterinaria y Biología Célula.

e Centro Nacional de Investigación Disciplinaria en Fisiología y Mejoramiento Animal, Instituto Nacional de Investigaciones Forestales, Agrícolas y Pecuarias. Km 1, carretera a Colón, C.P. 76280. Ajuchitlán, Colón, Querétaro, México.

Laboratorio de Análisis Térmico y Estructural de Alimentos, UIM, FES- Cuautitlán, UNAM. Carretera Cuautitlán-Teoloyucan Km 2.5, C.P. 54740. Cuautitlán Izcalli, Estado de México, México.
\end{abstract}

\section{RESUMEN}

La terneza es una característica importante para comercializar la carne de bovino, la cual se ha asociado a factores genéticos, como los polimorfismos de un solo nucleótido (SNPs). El objetivo de este trabajo fue analizar la distribución genotípica y alélica de los SNPs G530A, C357G, G1795A y G1181A para establecer asociación con la terneza. Se obtuvieron 90 muestras de músculo longissimus dorsi. Se determinó la terneza como fuerza de corte de WarnerBratzler. La genotipificación de los SNPs se realizó por PCRRFLP. Mediante un modelo lineal generalizado (GLM) se evaluó la asociación entre los SNPs y la terneza. El efecto de los alelos se determinó por análisis de sustitución alélica. Se determinó que el grupo genético influyó sobre la terneza $(p<0.05)$. Para los SNPs C530A y G1181A se obtuvo menor frecuencia para los genotipos $A A$, mientras que para los SNPs C357G y G1795A se observaron mayores frecuencias para los genotipos ancestrales CC y GG. No se encontró asociación genotípica o alélica entre los SNPs y la terneza. Esto evidencia la importancia de llevar a cabo análisis regionales de asociación entre SNPs y características de calidad en la búsqueda de su introducción en programas de selección asistida por marcadores moleculares.

Palabras clave: SNPs, PCR-RFLP, bovino, calidad de la carne, terneza

\section{ABSTRACT}

Tenderness, an important characteristic for marketing beef, has been associated with genetic factors, such as single nucleotide polymorphisms (SNPs). The aim of this work was to analyze the genotypic and allelic distribution of G530A, C357G, G1795A and G1181A SNPs to establish an association with meat tenderness. Ninety samples of longissimus dorsi muscle were obtained. Tenderness was determined as a
Warner-Bratzler shear force. SNPs genotyping was performed by PCR-RFLP. Using a generalized linear model (GLM), we evaluated the association between SNPs and tenderness. The effect of the alleles was determined by allelic substitution analysis. It was determined that the genetic group influenced tenderness ( $p<0.05$ ). For C530A and G1181A SNPs, a lower frequency was obtained for the AA genotypes, while for C357G and G1795A SNPs higher frequencies were observed for the ancestral CC and GG genotypes. We found no genotypic or allelic association between SNPs and tenderness. This shows the importance of carrying out regional analysis of association between SNPs and quality characteristics to introduce them favorably through marker-assisted selection programs.

Keywords: SNPs, PCR-RFLP, cattle, meat quality, tenderness.

\section{INTRODUCCIÓN}

La inconsistencia en la calidad de la carne es uno de los grandes retos que enfrenta la industria cárnica en México, causada por la falta de estrategias para producir ganado con características constantes y definidas (Bonilla et al., 2010). Los principales atributos que determinan la calidad de la carne son el color, la grasa intramuscular, el área del ojo del lomo y la palatabilidad. La palatabilidad, se relaciona con la terneza, la jugosidad y el sabor; de las anteriores, la terneza es la más importante para el consumidor como criterio de calidad (Li et al., 2013). La terneza es un atributo complejo, que se encuentra bajo el control de genes específicos y en existe evidencia que demuestra la existencia de una proporción significativa de la variabilidad fenotípica asociada a este atributo (Hocquette et al., 2007). La selección de los animales basándose en marcadores moleculares asociados a ésta característica puede ser una herramienta adicional a los esquemas normalmente utilizados en el mejoramiento genético animal (Enriquez et al., 2017). En México, se desconoce el

\footnotetext{
*Autor para correspondencia: Ana Elvia Sánchez Mendoza

Correo electrónico: anaesm@comunidad.unam.mx
} 
efecto en la implementación de estos marcadores genéticos. Sin embargo, se conoce al menos la frecuencia en la que las variantes favorables se presentan en algunas poblaciones y razas bovinas, lo que ayuda a inferir las ventajas de contar con esta información (Parra et al., 2011; Desgarennes et al., 2017).

En la actualidad se han identificado algunos SNPs como predictores de asociación con la terneza. La $\mu$-calpaína codificada por el gen CAPN1, es la principal enzima involucrada en la terneza de la carne debido a las bajas concentraciones de calcio que se requieren para su activación, dentro de este gen se encuentra el SNP G530A el cual provoca un cambio de adenosina por guanina en el codón 530 (GTC/ ATC), lo que resulta en el intercambio de una valina por isoleucina, esta alteración es relativamente conservada, aunque puede modificar la estabilidad de la proteína y su ensamblaje, lo que podría afectar su actividad proteolítica, por lo que este SNP se ha asociado fuertemente con la terneza (Page et al., 2002; Curi et al., 2009; Soria et al., 2010; Leal et al., 2015). La calpastatina es una enzima inhibidora de la actividad proteolítica de las calpaínas, se codifica por el gen CAST (Motter et al., 2009; Lenis et al., 2018), en donde se han reportado SNPs asociados con la terneza de la carne bovina (Motter et al., 2009; Chung y Davis, 2012; Desgarennes et al., 2017). Sin embargo, hay pocos estudios sobre las variaciones de secuencias o cambios en las regiones reguladoras potenciales que pudieran explicar las diferencias fenotípicas en la calidad de la carne. Juszczuk-Kubiak et al. (2009), sugieren que el SNP C357G localizado en el exón 1u asociado al promotor III, podría tener influencia sobre la expresión de este gen y repercutir en rasgos importantes de la calidad como la terneza. Las múltiples funciones en los ensambles de proteínas de la línea Z y la banda I atribuidas a la proteína Miopaladina codificada por el gen MYPN, demuestran una asociación de los SNPs de este gen con la terneza. Jiao et al. (2010) estudiaron el SNP G1795A y su asociación con ciertas características fenotípicas, como la pérdida de retención de agua, lo que favorece la disminución de la terneza de la carne. Por otro lado, MYPN se une a la proteína cardíaca de repetidos de anquirina (CARP) (Bang et al., 2001; Mestroni, 2009). La sobreexpresión de la región de unión CARP con miopaladina podría provocar una interrupción severa de los componentes sarcoméricos, lo cual sugiere que la organización miofibrilar está relacionada con la expresión génica en el músculo a través de la interacción de miopaladina con CARP (Bang et al., 2001; Knöll, Hoshijima y Chien, 2002), esto convierte al gen de miopaladina y sus SNPs como posibles candidatos asociados a terneza. El gen receptor gama coactivador 1-a, activado por el proliferador de peroxisoma (PPARGC1a) tiene funciones en la regulación del metabolismo de la energía oxidativa en el músculo esquelético (Eivers et al., 2012) además de mediar la expresión de genes involucrados en la adipogénesis provocando un aumento en el contenido de grasa intramuscular lo que sugiere una influencia potencial sobre la terneza (Lin, Handschin y Spiegelman, 2005; Zou et al., 2014). La presencia del SNP G1181A en este gen, provoca la sustitución del aminoácido serina por asparagina, lo cual interrumpe un sitio de fosforilación de la caseína kinasa II, lo que puede afectar importantes rasgos como la terneza (Soria et al., 2009).

Los SNPs analizados en el presente trabajo se han descrito ampliamente en diferentes grupos genéticos y zonas geográficas a nivel mundial, por lo que estos SNPs han sido propuestos como importantes marcadores asociados a la terneza para su implementación en programas de Selección asistida por marcadores (SAM).

La identificación de la presencia de estos SNPs en los programas de selección asistida por marcadores moleculares (SAM) depende de la validación de sus efectos sobre los rasgos de interés en diferentes condiciones de manejo y ambiente, por lo que su evaluación es muy importante para asegurar la eficacia en su implementación (Van Eenennaam et al., 2007; Parra et al., 2011).

Por lo anterior el objetivo de este trabajo fue analizar la distribución genotípica y alélica de los SNPs G530A, C357G, G1795A y G1181A para establecer su asociación con la terneza de carne de bovinos del Estado de Veracruz.

\section{MATERIALES Y MÉTODOS Animales y toma de muestra}

Se obtuvieron 90 muestras de $5 \mathrm{~cm}$ de espesor del músculo longissimus dorsi (entre la 12va y 13va vértebra lumbar), provenientes de toros no castrados, alojados en corrales de una unidad comercial de finalización del sur de Veracruz, con un peso promedio de $470 \pm 20 \mathrm{~kg}$ y edad de 18 a 21 meses, los cuales fueron faenados bajo los lineamientos de la NOM-033-SAG/ZOO-2014, en el rastro de Tipo Inspección Federal 35, ubicado en el Km 2.5, carretera Paso San Juan, Veracruz. Las muestras se clasificaron de acuerdo a su grupo genético, observando las principales características fenotípicas reportadas por Rubio et al. (2013), en Bos taurus, Bos indicus y la cruza Bos taurus $x$ Bos indicus.

A cada corte obtenido se le retiró una muestra de $250 \mathrm{mg}$ de tejido, estas muestras se depositaron en tubos con $1 \mathrm{~mL}$ de Trizol $^{\circledast}$ (Invitrogen, Carlsbad, Ca, EE.UU.) y se mantuvieron a $-20^{\circ} \mathrm{C}$ hasta realizar los análisis moleculares correspondientes.

\section{Determinación de fuerza de corte}

La fuerza de corte de Warner-Bratzler de las muestras cocidas se determinó de acuerdo con el método descrito por la AMSA (2015). Inicialmente se descongelaron a $4^{\circ} \mathrm{C}$ durante 24 h, se les retiró la grasa y el tejido conectivo, a continuación fueron sometidas a un proceso de cocción en una parrilla eléctrica (Westinghouse, Pittsburgh, PA, EE.UU). La superficie de la parrilla se calentó a $200^{\circ} \mathrm{C}$ antes de la cocción. Las muestras se cocinaron hasta que alcanzaron una temperatura interna de $70^{\circ} \mathrm{C}$ verificando la temperatura en el centro geométrico de cada muestra utilizando un termopar tipo T de bayoneta (Testo, 0602 1081, Barcelona, Esp). Al término de la cocción, se colocaron individualmente en bolsas de plástico 
y se enfriaron a temperatura ambiente durante 30 minutos, a continuación se realizaron seis cortes cilíndricos en forma paralela a la orientación longitudinal de las fibras musculares con un dispositivo de extracción manual (sacabocado). A cada cilindro se le cortó con una cuchilla Warner-Bratzler del analizador de textura TA-XT plus (Stable Micro Systems, Godalming, LDN, UK) que operó a una velocidad de prueba de 2 $\mathrm{mm} / \mathrm{s}$. Los valores de la fuerza de corte se registraron como el esfuerzo máximo expresado en $\mathrm{kg}$, el valor promedio de los cortes se utilizó para el análisis estadístico.

\section{Identificación de SNPs}

El ADN genómico se extrajo de las muestras de carne empleando el protocolo propuesto por Sambrook, J. (2012) el cual se basa en la disgregación del tejido con $1250 \mu \mathrm{L}$ de solución de lisis y $7 \mu \mathrm{L}$ de proteinasa $\mathrm{k}$ (Bioline, Memphis, TE, EE.UU), seguido de la extracción de proteínas y polisacáridos con $250 \mu \mathrm{L}$ de fenol-cloroformo-alcohol isoamílico (SigmaAldrich, St. Louis, MO, EE.UU.) y finalmente la precipitación de ADN con $1000 \mu \mathrm{L}$ de etanol frio (Sigma-Aldrich, St. Louis, MO, EE.UU.)

La identificación de los SNPs, se llevó a cabo mediante primers específicos usando las secuencias reportadas en GenBank de acuerdo con los siguientes números de acceso: calpaína AF248054, calpastatina AH014526.1 y miopaladina NC_007329. En el caso del gen PPARG1A se utilizaron los primers reportados por Soria et al. (2009) (Tabla 1). La mezcla de reacción para la PCR se preparó bajo las siguientes condiciones en un volumen total de $25 \mu \mathrm{L}: 0.5 \mu \mathrm{L}$ de cada primer, $10 \mu \mathrm{L}$ de agua libre de nucleasas, $60 \mathrm{ng}$ de ADN y $12.5 \mu \mathrm{L}$ de la mezcla Master mix (Promega Corporation, Fitchburg, WI), la cual contiene 50 unidades $/ \mathrm{mL}$ de Taq ADN polimerasa, $400 \mu \mathrm{M}$ de cada dNTP y $3 \mathrm{mM}$ de $\mathrm{MgCl}_{2}$. Las condiciones de amplificación de cada gen fueron calculadas de acuerdo a las recomendaciones del kit de Promega, considerando la temperatura de hibridación de cada pareja de primers. Los productos amplificados se analizaron por electroforesis en geles de agarosa al $1.5 \%$ con $0.4 \mu \mathrm{g}$ de bromuro de etidio y visualizados en un transiluminador de luz ultravioleta (Bio Imaging Systems, Dún Laoghaire, Dublin).

\section{Genotipificación de SNPs}

La genotipificación de cada SNPS se realizó mediante RFLP (polimorfismo de longitud de fragmentos de restricción), digiriendo los productos de PCR con enzimas de restricción específicas. Las enzimas empleadas fueron: Ava II (G530A), Hae III (G1795A), Taq I (C357G) y Bst I (G1181A). Las digestiones se realizaron en reacciones de $10 \mu \mathrm{L}$ compuestas por $5 \mu \mathrm{L}$ del producto de PCR, $3.6 \mu \mathrm{L}$ de agua libre de nucleasas, $1 \mu \mathrm{L}$ de solución tampón y $0.4 \mu \mathrm{L}$ de enzima, la temperatura empleada fue la recomendada por el fabricante (New England Biolabs, Ipswich, MA). Los patrones de digestión se visualizaron en geles de acrilamida:bis al 12\% (29:1, SigmaAldrich, St. Louis, MO, EE.UU.), los cuales fueron teñidos con bromuro de etidio $(10 \mathrm{mg} / \mathrm{mL}$, Gene Choice, Inc., Frederick, MD, EE.UU.).
Tabla 1. Primers empleados en la identificación de los SNPS.

Table 1. Primers used for SNPs identification.

\begin{tabular}{|c|c|c|c|c|}
\hline GEN & SNP & Secuencia & $\begin{array}{l}\mathrm{Tm} \\
\left({ }^{\circ} \mathrm{C}\right)\end{array}$ & $\begin{array}{c}\text { Amplificado } \\
\text { (pb) }\end{array}$ \\
\hline Calpaína & $A>G$ & $\begin{array}{l}\text { F: 5'-TCTGCAGAGAGCTG } \\
\text { GATGAC-3' } \\
\text { R: 5'-TGCTGGGCTAGAGAC } \\
\text { CAAGAC-3' }\end{array}$ & 56 & 119 \\
\hline Calpastatina & $G>C$ & $\begin{array}{l}\text { F: 5'-GCTAGCCAAGGCTACA } \\
\text { TCTCC-3' } \\
\text { R: 5'-AAGCATGCGAACTG } \\
\text { AAACGC-3' }\end{array}$ & 59 & 101 \\
\hline Miopaladina & $A>G$ & $\begin{array}{l}\text { F: 5'-TCTGTACTTTGGTCATT } \\
\text { GCTGAG-3' } \\
\text { R: 5'-CCCTGGTCTAGACGG } \\
\text { TTATGG-3' }\end{array}$ & 55 & 150 \\
\hline PPARGC $1 A^{*}$ & $A>G$ & $\begin{array}{l}\text { F: 5'-TCAGCAAGACCTCTGTGCTCA- } \\
\text { GCA-3' } \\
\text { R: 5'-TGCTCACCTCCGCGGTCTCT-3' }\end{array}$ & 62 & 255 \\
\hline
\end{tabular}

*Secuencias reportadas por Soria et al., (2009).

\section{Análisis estadístico}

La prueba de equilibrio de Hardy-Weinberg (HW) se realizó para cada SNP, la cual determinó cuales frecuencias deben observarse en la población para cada genotipo en función de las frecuencias de los alelos (Iniesta, Guinó y Moreno, 2005). Las frecuencias alélicas y genotípicas fueron calculadas de acuerdo a Iniesta, Guinó y Moreno, (2005). El equilibrio HW se determinó mediante la prueba de $X^{2}$ ( $\mathrm{Ji}$ cuadrada) usando el programa SPSS (26.0, 2019).

La prueba de asociación entre los genotipos y los rasgos de interés se llevó a cabo mediante el método de mínimos cuadrados y el procedimiento del modelo lineal generalizado (GLM) a través del programa SPSS $(26.0,2019)$ (Curi et al., 2010; Chung y Davis 2012; Valencia y Zuluaga 2012; Enríquez et al., 2017). El modelo utilizado para ajustar las variables cuantitativas de interés incluyó el efecto fijo del genotipo de cada SNP y el efecto fijo del grupo genético y fue el siguiente:

$$
\begin{aligned}
& Y_{i j k l m}=\mu+G 1_{i}+G 2_{j}+G 3_{k}+G 4_{1}+G G_{m}+\varepsilon_{i j k l m} \\
& \text { Donde: } \\
& \mathrm{Y}_{\mathrm{ijklm}}=\text { Variable dependiente (WBSF) } \\
& \mu=\text { media global. } \\
& \mathrm{G} 1_{\mathrm{i}}=\text { efecto fijo del i-ésimo genotipo de G530A. } \\
& \text { G2j = efecto fijo del j- ésimo genotipo de C357G. } \\
& \mathrm{G}_{\mathrm{k}}=\text { efecto fijo del k- ésimo genotipo de G1795A. } \\
& \text { G4 } \text { I= efecto fijo del I- ésimo genotipo de G1181A. } \\
& \mathrm{GGm}=\text { efecto fijo del } \mathrm{m} \text { - ésimo grupo genético. } \\
& \varepsilon_{\mathrm{ijklm}}=\text { error aleatorio. }
\end{aligned}
$$

Los efectos fijos que fueron significativos se analizaron mediante una prueba de comparación de medias de Tukey a través del programa SPSS $(26.0,2019)$ para determinar las diferencias entre los niveles de cada uno de los efectos.

El efecto de los alelos de cada SNP sobre la terneza se determinó mediante un análisis de sustitución alélica, para lo cual se transformó la información de la genotipificación en información cuantitativa asignándole valores de 0 al genotipo ancestral, 1 al genotipo heterocigoto y 2 al genotipo de la variante genética para cada SNP. Este análisis se realizó 
a través de una regresión lineal simple en el programa SPSS (26.0, 2019) (Valencia y Zuluaga 2012; Arango, Echeverri y López 2014) aplicando el siguiente modelo:

$$
Y=\beta_{0}+\beta_{1} X_{i}
$$

Donde:

Y: Variable dependiente (WBSF).

$\beta_{0}$ : Intercepto.

$\beta_{1}$ : Coeficiente de regresión estimado para la sustitución alélica de cada SNP (G530A, C357G, G1795A y G1181A).

Xi: Número de alelos de la variante genética $(0,1$ o 2).

\section{RESULTADOS}

\section{Fuerza de corte (WBSF)}

La fuerza de corte presentó una media global de 6.40 $\mathrm{kg}$, el análisis estadístico mostró que el grupo genético es un factor que influye significativamente sobre el rasgo de calidad. Los resultados obtenidos cuando se analizaron por medio de la prueba de Tukey mostraron una diferencia significativa de la terneza entre los grupos genéticos Bos taurus V.S Bos indicus $(\mathrm{p}<0.05)$, en donde se observó que la carne de Bos taurus fue más tierna $(6.083 \pm 0.124 \mathrm{~kg})$ al compararla con la de Bos indicus $(6.861 \pm 0.097 \mathrm{~kg})$, por otro lado, en la carne procedente de la cruza, no se observaron diferencias significativas al compararlas con los otros dos grupos genéticos que fueron estudiados (Tabla 2).

Tabla 2. Efecto del grupo genético sobre la fuerza de corte Warner-Bratzler (WBSF) en carne bovina.

Table 2. Genetic group effect on Warner-Bratzler shear force (WBSF) in beef.

\begin{tabular}{lcc}
\hline \multicolumn{1}{c}{ Grupo genético } & N & $\begin{array}{c}\text { WBSF }(\mathbf{k g}) \\
\text { Media } \pm \text { ESM }\end{array}$ \\
\hline Bos taurus & 31 & $6.083 \pm 0.124^{\mathrm{b}}$ \\
Bos taurus x Bos indicus & 30 & $6.405 \pm 0.093^{\mathrm{ab}}$ \\
Bos indicus & 29 & $6.861 \pm 0.097^{\mathrm{a}}$ \\
\hline
\end{tabular}

Comparación de medias por la prueba de Tukey. Literales diferentes indican diferencias significativas $(p<0.001)$.

Frecuencias genotípicas y alélicas de los SNPs G530A (calpaína), C357G (calpastatina), G1795A (miopaladina) y G1181A (PPARGC1a)

Los resultados de las frecuencias genotípicas y alélicas determinadas para cada SNP se observan en la tabla 3.

El equilibrio de Hardy-Weinberg se cumplió en tres SNPs; G530A, G1795A y G1181A, mientras que el SNP de calpastatina (C357G) no cumplió con el criterio de equilibrio $(p \leq 0.05)$.

Los tres genotipos fueron detectados en todos los SNPs estudiados. Para el SNP de calpaína, el genotipo favorable para terneza (AA) tuvo una baja frecuencia (0.011). El genotipo GG fue el dominante, ya que presentó una alta frecuencia (0.767).

El SNP G1181A del gen PPARGC1 mostró una baja frecuencia para el genotipo $A A$, correspondiente a la variante genética (0.033), en el genotipo GG este SNP presentó una mayor frecuencia (0.522).
Tabla 3. Frecuencias genotípicas y alélicas para los SNPs. Table 3. Genotypic and allelic frequencies for SNPS.

\begin{tabular}{lcccccc}
\hline SNP & Genotipo & N & $\begin{array}{c}\text { Frecuencia } \\
\text { genotípica }\end{array}$ & Alelo & $\begin{array}{c}\text { Frecuencia } \\
\text { Alélica }\end{array}$ & $\begin{array}{c}\text { Equilibrio } \\
\text { HW }\end{array}$ \\
\hline G530A & AA & 1 & 0.011 & $\mathrm{~A}$ & 0.122 & \\
calpaína & GG & 69 & 0.767 & & & $* *$ \\
C357G & AG & 20 & 0.222 & G & 0.878 & \\
calpastatina & GG & 7 & 0.078 & G & 0.183 & \\
& GC & 64 & 0.711 & & & NE \\
G1795A & AA & 21 & 0.233 & A & 0.517 & \\
miopaladina & GG & 18 & 0.200 & & & $* *$ \\
& AG & 51 & 0.567 & G & 0.483 & \\
G1181A & AA & 3 & 0.033 & A & 0.256 & \\
PPARGC1a & GG & 47 & 0.522 & & & $* *$ \\
& AG & 40 & 0.444 & G & 0.744 & \\
\hline
\end{tabular}

$H W=$ Equilibrio de Hardy-Weinberg; ${ }^{* *}(p<0.01)$; NE (no equilibrio de HW) $=(p>0.05)$.

En el SNP C357G (calpastatina) la mayor frecuencia se observó en el genotipo CC (0.711), mientras que la menor frecuencia la presentó el genotipo GG (0.078).

Por último, para el SNP G1795A de miopaladina, el genotipo heterocigoto AG presentó una alta frecuencia (0.567), la menor frecuencia de este SNP la presentó el genotipo ancestral GG (0.200).

Efecto de los SNPs G530A (calpaína), C357G (calpastatina), G1795A (miopaladina) y G1181A (PPARGC1A) sobre la terneza

El factor del genotipo AA del SNP G530A se descartó para el análisis estadístico, debido a la baja frecuencia mostrada en la población.

Los resultados de la aplicación del modelo de asociación de los SNPs con la terneza no mostraron diferencias significativas ( $p>0.05$ ) lo cual sugiere que ninguno de los SNPs influye sobre la terneza de la carne de los animales analizados (tabla 4).

Efecto de la sustitución alélica de los SNPs G530A (calpaína), C357G (calpastatina), G1795A (miopaladina) y G1181A (PPARGC1A) sobre la terneza

Los resultados del análisis de regresión lineal entre los alelos de cada SNP y las variaciones de la terneza se muestran en la tabla 5. De acuerdo con el valor de $\beta_{1}$, la terneza se incrementa $0.261 \mathrm{~kg}$ con la presencia del alelo A para el SNP G530A, de igual forma para el SNP G1181A la presencia del alelo A incrementa la terneza $0.091 \mathrm{~kg}$. Mientras que para los SNPs C357G y G1795A los alelos de la variante genética repercutieron en una disminución de la terneza de $0.021 \mathrm{~kg}$ y $0.081 \mathrm{k}$, respectivamente. El análisis estadístico mostró que para todos los SNPs el efecto de la sustitución alélica no fue significativo ( $p>0.05$ ), estos resultados sugieren que la presencia del alelo de la variante genética no tiene un efecto sobre la terneza de la carne de la población analizada. 
Tabla 4. Efecto de los SNPs G530A, C357G, G1795A y G1181A sobre la fuerza de corte Warner-Bratzler (WBSF) en carne bovina.

Table 4. Effect of G530A, C357G, G1795A and G1181A SNPs on Warner-Bratzler shear force (WBSF) in beef.

\begin{tabular}{|c|c|c|c|c|}
\hline SNP & Genotipo & $\mathbf{N}$ & & $\begin{array}{c}\text { WBSF (kg) } \\
\text { Media } \pm \text { ESM }\end{array}$ \\
\hline \multirow{4}{*}{$\begin{array}{l}\text { G530A } \\
\text { Calpaína }\end{array}$} & & & $p=$ & 0.537 \\
\hline & $A A$ & - & & - \\
\hline & GG & 69 & & $6.4633 \pm 0.0724$ \\
\hline & AG & 20 & & $6.278 \pm 0.163$ \\
\hline \multirow{4}{*}{$\begin{array}{l}\text { C357G } \\
\text { Calpastatina }\end{array}$} & & & $p=$ & 0.989 \\
\hline & GG & 7 & & $6.603 \pm 0.186$ \\
\hline & $\mathrm{CC}$ & 64 & & $6.441 \pm 0.075$ \\
\hline & GC & 19 & & $6.228 \pm 0.180$ \\
\hline \multirow{4}{*}{$\begin{array}{l}\text { G1795A } \\
\text { Miopaladina }\end{array}$} & & & $p=$ & 0.395 \\
\hline & $A A$ & 21 & & $6.371 \pm 0.135$ \\
\hline & GG & 18 & & $6.540 \pm 0.149$ \\
\hline & AG & 51 & & $6.378 \pm 0.092$ \\
\hline \multirow{4}{*}{$\begin{array}{l}\text { G1181A } \\
\text { PPARGC1a }\end{array}$} & & & $p=$ & 0.803 \\
\hline & AA & 3 & & $6.890 \pm 0.040$ \\
\hline & GG & 47 & & $6.401 \pm 0.110$ \\
\hline & AG & 40 & & $6.384 \pm 0.088$ \\
\hline
\end{tabular}

No existen diferencias significativas, $\mathrm{p}>0.05$.

Tabla 5. Efecto de la sustitución alélica de cada SNP sobre sobre la fuerza de corte Warner-Bratzler; I (intercepto), $\beta_{1}$ (coeficiente de regresión entre los genotipos de los diferentes SNPs y la fuerza de corte).

Table 5. Allelic substitution effect of each SNPs on the Warner-Bratzler shear force (WBSF); I (intercept), $\beta_{1}$ (regression coefficient between the genotypes of the different SNPs and the Warner-Bratzler).

\begin{tabular}{lccc}
\hline SNP & $\mathbf{I}$ & $\boldsymbol{\beta}_{1}$ & $\mathbf{p}$ \\
\hline $\begin{array}{l}\text { G530A } \\
\text { (calpaina) }\end{array}$ & 6.473 & 0.261 & 0.079 \\
$\begin{array}{l}\text { C357G } \\
\text { (calpastatina) }\end{array}$ & 6.417 & -0.021 & 0.847 \\
$\begin{array}{l}\text { G1795A } \\
\text { (miopaladina) }\end{array}$ & 6.493 & -0.081 & 0.432 \\
$\begin{array}{l}\text { G1181A } \\
\text { (PPARGC1a) }\end{array}$ & 6.363 & 0.091 & 0.452 \\
\hline
\end{tabular}

No existen diferencias significativas, $\mathrm{p}>0.05$.

\section{DISCUSIÓN}

La validación de los SNPs y su relación con la terneza de la carne es importante para el desarrollo e implementación de los programas de mejoramiento genético en los bovinos.

Los resultados del factor fijo del grupo genético mostraron tener una influencia sobre la terneza, ya que se observaron en los grupos genéticos estudiados diferencias en este rasgo y los cuales son similares con los reportados por otros autores (Casas et al., 2006; Soria et al., 2009; Page et al., 2002, De Souza et al., 2017), quienes sugieren que además del factor grupo genético esas diferencias se pueden atribuir a SNPs presentes en genes que desempeñan un papel determinante durante el proceso de tenderización.

Las frecuencias genotípicas para un gen pueden ser afectadas por diferentes fuerzas evolutivas como; mutación, deriva genética, selección natural, selección dirigida de rasgos fenotípicos de producción, migración, las cuales pueden aumentar la diferenciación genética de las poblaciones (Eguiarte et al., 2013). Los resultados de la prueba de equilibrio de Hardy-Weinberg, no se cumplió para el SNP de calpastatina (C357G), lo cual podría deberse a los factores anteriormente mencionados.

El genotipo AA del SNP G530A se ha asociado con la terneza por Casas et al. (2006), Van Eenennaam et al. (2007), Allais et al. (2011) y Li et al. (2010), quienes reportaron que el ganado con este genotipo presenta carne más blanda en comparación con animales con genotipos AG y GG, sin embargo, en este estudio el genotipo AA mostró una baja frecuencia ya que sólo se identificó en un animal del grupo genético Bos taurus, mientras que el genotipo GG fue el predominante para todos los grupos genéticos, estos resultados son similares a los reportados por Casas et al. (2005), quienes mencionaron la ausencia del alelo $\mathrm{A}$ en animales Bos indicus.

Por otro lado, los resultados no mostraron asociación del SNP G530A con la terneza, lo cual concuerda con lo reportado por Curi et al. (2009), quienes mencionaron que no existe una asociación del SNP G530A con la terneza en diferentes grupos genéticos al encontrar una baja frecuencia del genotipo AA y la predominancia del genotipo GG.

Juszczuk-Kubiak et al. (2009) encontró que el SNP C357G se localiza en la región promotora del gen CAST y sugirieron que posiblemente esté tenga participación con la terneza de la carne, en ese trabajo se encontró la presencia de este SNP, sin embargo, los resultados demuestran que no tiene un efecto sobre la terneza de la carne. Por otro lado, es importante destacar que este es el primer reporte de la evaluación de este SNP en ganado de producción nacional, en donde se demuestra que no tiene una asociación con la terneza. Sin embargo, los datos de las distribuciones de frecuencias alélicas y genotípicas determinadas en esta población contribuyen al conocimiento de SNPs asociados a terneza y podrían ser de utilidad para otros estudios similares.

Los resultados obtenidos en este trabajo con respecto al SNP G1795A mostraron una frecuencia baja del genotipo $A A$, mientras que la mayor frecuencia se observó para el genotipo heterocigoto AG. Con respecto a las frecuencia alélicas, el alelo $\mathrm{A}$ mostró la mayor frecuencia, sin embargo, mediante el análisis de sustitución alélica se demostró que la presencia del alelo $A$ no influyó significativamente sobre la terneza ( $p>0.05)$, estos resultados difieren a lo reportado por Jiao et al. (2010) quienes analizaron siete subpoblaciones de ganado Bos taurus y encontraron una mayor frecuencia del alelo $A$ en comparación con el alelo $G$, asociando al alelo A con el área del ojo del lomo y la capacidad de retención de agua en ganado bovino de la subespecie Bos taurus, lo que favorece a la terneza, además de establecer de que este SNP 
podría considerarse como característico de ganado Bos taurus. Por el contrario, en este trabajo la mayor frecuencia del genotipo AA se observó en el grupo genético Bos indicus, por lo cual se sugiere que para la población analizada, la segregación del alelo favorable para las características de la calidad de la carne no es exclusivo de ningún grupo genético.

La presencia del genotipo AA del SNP G11181 sólo se encontró en el grupo genético Bos indicus lo cual coincide con lo reportado por Soria et al. (2009), quienes propusieron al SNP G1181 como candidato para un panel de marcadores moleculares asociados a los rasgos de la calidad de la carne en especial en el ganado Bos indicus, ya que la presencia del alelo $A$ no fue identificado en el ganado Bos taurus. Por otro lado, el análisis de asociación con la terneza determinó que este SNP no presentó ninguna asociación con este rasgo, la ausencia de esta asociación se puede atribuir en parte, a que la región en donde se localiza este polimorfismo es una región no conservada entre grupos.

\section{CONCLUSIONES}

Los SNPs analizados no presentaron asociación alélica o genotípica con el rasgo de terneza, estos resultados refuerzan la importancia de hacer estos estudios de asociación de forma regional ya que la distribución de los genotipos entre las diversas subespecies que se comercializan es muy variable dependiendo del origen del ganado, de las diferentes poblaciones coexistentes y de la mezcla de distintas razas; lo anterior, aunando a mejoras en los registros de los parámetros productivos y reproductivos en las explotaciones ganaderas, contribuirá sin duda a una implementación exitosa de los programas de manejo asistido por marcadores moleculares, lo que dará como resultado una mejora en la selección de la calidad de la carne en beneficio a los productores.

\section{AGRADECIMIENTOS}

Al programa de Posgrado en Ciencias de la Salud y la Producción Animal de la FES-Cuautitlán, UNAM, al Consejo Nacional de Ciencia y Tecnología (CONACYT), al M en C. Manuel Andrés González Toimil de la FES-Cuautitlán, UNAM, al Dr. Pedro Garcés Yépez y al Dr. Celis e Ing. Celis del rancho Santa Rita.

\section{FINANCIAMIENTO}

Este estudio fue parcialmente financiado por la UNAM, FES- Cuautitlán a través de sus programas PIAPI1837: "Aplicación de herramientas moleculares en el área agroalimentaria y diagnóstico de enfermedades mitocondriales", PAPIIT IN226419: "Asociación del fondo genético mitocondrial en enfermedades mitocondriales de la población mexicana" y la beca escolar otorgada a la autora a través del Consejo Nacional de Ciencia y Tecnología (CONACYT), ID 422754.

\section{REFERENCIAS}

Allais, S., Journaux, L., Levéziel, H., Payet-Duprat, N., Raynaud, P., Hocquette, J. F., Lepetit, J., Rousset, S., Denoyelle, C., Bernard-
Capel C., Renand, G. 2011. Effects of polymorphisms in the calpastatin and $\mu$-calpain genes on meat tenderness in 3 French beef breeds. Journal of Animal Science. 89 (1): 1-11. Doi: $10.2527 /$ jas.2010-3063.

AMSA. 2015. Research Guidelines for Cookery, Sensory Evaluation and Instrumental Tenderness Measurements of Meat. American Meat Science Association National Live Stock and Meat Board, Chicago, IL. USA.

Arango, G., J., Echeverri, Z., J., López, H., A. 2014. Asociación del gen de la hormona de crecimiento bovino con parámetros reproductivos en ganado Holstein. Revista MVZ Córdoba. 19 (3): 4249-4258.

Bang, M. L., Mudry, R. E., McElhinny, A. S., Trombitás, K., Geach, A. J., Yamasaki, R., Sorimachi, H., Granzier, H., Gregorio, C., Labeit, S. 2001. Myopalladin, a novel 145-kilodalton sarcomeric protein with multiple roles in Z-disc and I-band protein assemblies. Journal of Cell Biology. 153 (2): 413-427. Doi: 10.1083/jcb.153.2.413.

Bonilla, C. A., Rubio, M. S., Sifuentes, A. M., Parra, G. M., Arellano, V. W., Méndez, M. R.D., Berruecos, J. M., Ortiz, R. 2010. Association of CAPN1 316, CAPN1 4751 and TG5 markers with bovine meat quality traits in Mexico. Genetics and Molecular Research: GMR. 9 (4): 2395-2405. Doi: 10.4238/ vol9-4gmr959.

Casas, E., White, S. N., Riley, D. G., Smith, T. P. L., Brennemant, R. A., Olson, T. A., Johnson, D. D., Coleman, S. W., Bennett, G. L., Chase, C. C. 2005. Assessment of single nucleotide polymorphisms in genes residing on chromosomes 14 and 29 for association with carcass composition traits in Bos indicus cattle. Journal of Animal Science. 83 (1): 13-19. Doi: 10.2527/2005.83113x.

Casas, E., White, S. N., Wheeler, T. L., Shackelford, S. D., Koohmaraie, M., Riley, D. G., Chase, C. C., Johnson, D. D., Smith, T. P. L. 2006. Effects of calpastatin and $\mu$-calpain markers in beef cattle on tenderness traits. Journal of Animal Science. 84 (3): 520-525. Doi: 10.2527/2006.843520x.

Chung, H., Davis, M. 2012. Effects of genetic variants for the calpastatin gene on calpastatin activity and meat tenderness in Hanwoo (Korean cattle). Meat Science. 90 (3): 711-714. Doi: 10.1016/j.meatsci.2011.10.017.

Curi, R. A., Chardulo, L. A. L., Giusti, J., Silveira, A. C., Martins, C. L., De Oliveira, H. N. 2010. Assessment of GH1, CAPN1 and CAST polymorphisms as markers of carcass and meat traits in Bos indicus and Bos taurus-Bos indicus cross beef cattle. Meat Science, 86(4), 915-920. Doi:10.1016/j.meatsci.2010.07.016.

Curi, R. A., Chardulo, L. A. L., Mason, M. C., Arrigoni, M. D. B., Silveira, A. C., \& De Oliveira, H. N. 2009. Effect of single nucleotide polymorphisms of CAPN1 and CAST genes on meat traits in Nellore beef cattle (Bos indicus) and in their crosses with Bos taurus. Animal Genetics. 40 (4): 456-462. Doi: 10.1111/j.1365-2052.2009.01859.x.

De Souza, R. R.T., Chizzotti, M. L., Vital, C. E., Baracat, P. M. C., Barros, E., Busato, C. K., Aparecido, G. R., Machad, L.M., Martins, S. T. (2017). Differences in beef quality between Angus (Bos taurus taurus) and Nellore (Bos taurus indicus) cattle through a proteomic and phosphoproteomic approach. PLoS ONE, 12(1). DOI: 10.1371/journal.pone.0170294.

Desgarennes, A., C. M., Del Moral. V., S., Meza, V., V. M., Peña, C., J. M., Zárate, M., J. P., Abad, Z., J. 2017. Estimación de las frecuencias alélicas y genotípicas de los genes CAPN1 Y CAST asociados a la calidad de la carne en bovinos de 
la Cuenca del Papaloapan. Nova Scientia, 9(19), 211. Doi: 10.21640/ns.v9i19.996.

Eguiarte, L. E., Aguirre, J. A., Jardón, L., Aguirre, E., Souza, V. 2013. Genómica de Poblaciones: Nada en Evolución va a tener sentido si no es a la luz de la Genómica, y nada en Genómica tendrá sentido si no es a la luz de la Evolución. TIP Revista Especializada en Ciencias Químico-Biológicas. 16 (1): 42-56. Doi: 10.1016/s1405-888x(13)72077-1.

Eivers, S. S., McGivney, B. A., Gu, J., MacHugh, D. E., Katz, L. M., Hill, E. W. 2012. PGC-1a encoded by the PPARGC1A gene regulates oxidative energy metabolism in equine skeletal muscle during exercise. Animal Genetics. 43 (2): 153-162. Doi: 10.1111/j.1365-2052.2011.02238.x.

Enriquez, V., C. E., Pereira, G. L., Malheiros, J. M., de Vasconcelos Silva, J. A. I. I., Albuquerque, L. G., de Oliveira, H. N., Loyola, C. L. A., Curi, R. A. 2017. Effect of the g.98535683A > G SNP in the CAST gene on meat traits of Nellore beef cattle (Bos indicus) and their crosses with Bos taurus. Meat Science. 123: 64-66. Doi: 10.1016/j.meatsci.2016.09.003.

Hocquette, J. F., Lehnert, S., Barendse, W., Cassar-Malek, I., Picard, B. 2007. Recent advances in cattle functional genomics and their application to beef quality. Animal. 1 (1): 159-173. Doi: 10.1017/S1751731107658042.

IBM Corp. Released 2019. IBM SPSS Statistics for Windows, Version 26.0. Armonk, NY: IBM Corp.

Iniesta, R., Guinó, E., Moreno, V. 2005. Statistical analysis of genetic polymorphisms in epidemiological studies. Gaceta Sanitaria. 19 (4): 333-341. Doi: 10.1157/13078029.

Jiao, Y., Zan, L. S., Liu, Y. F., Wang, H. B., Guo, B. L. 2010. A novel polymorphism of the MYPN gene and its association with meat quality traits in Bos taurus. Genetics and Molecular Research: GMR. 9 (3): 1751-1758. Doi: 10.4238/vol93gmr906.

Juszczuk-Kubiak, E., Flisikowski, K., Wicinska, K., Poloszynowicz, J., \& Rosochacki, S. 2009. Identification of the new polymorphisms in the promoter region of the CAST gene in cattle. Meat Science. 82 (2): 278-283. Doi: 10.1016/j. meatsci.2009.01.001.

Knöll, R., Hoshijima, M., Chien, K. R. 2002. Z-line proteins: Implications for additional functions. European Heart Journal. Supplement 4 (1). Doi: 10.1016/S1520 765X(02)90105-7.

Leal-Gutiérrez, J. D., Jiménez-Robayo, L. M., Ariza, M., Manrique, C., López, J., Martínez, C., Pinilla, Y., Castro, S., García, N., Bedoya, C., Jiménez, A. 2015. Polimorfismos de los genes CAPN1, CAST, DES, PRKAG3 y RYR1 asociados a la capacidad de retención de agua en crudo y cocinado en carne de bovino en cruces Bos indicus y Bos taurus en Colombia. Archivos de Zootecnia. 64 (245): 29-35. Doi: 10.21071/ az.v64i245.371.

Lenis, C., Ramos, L., Hernández Herrera, D., Álvarez, L. 2018. Polimorfismos de los genes calpaína y calpastatina en el ganado criollo colombiano Hartón del Valle. Revista de Investigaciones Veterinarias Del Perú. 29 (3): 818. Doi: 10.15381/rivep.v29i3.14003.

Li, J., Zhang, L. P., Gan, Q. F., Li, J. Y., Gao, H. J., Yuan, Z. R., Gao, X., Chen, J.B., Xu, S. Z. 2010. Association of CAST gene polymorphisms with carcass and meat quality traits in chinese commercial cattle herds. Asian-Australasian Journal of Animal Sciences. 23 (11): 1405-1411. Doi: 10.5713/ ajas.2010.90602.
Li, X., Ekerljung, M., Lundström, K., Lundén, A. 2013. Association of polymorphisms at DGAT1, leptin, SCD1, CAPN1 and CAST genes with color, marbling and water holding capacity in meat from beef cattle populations in Sweden. Meat Science. 94 (2): 153-158. Doi: 10.1016/j.meatsci.2013.01.010.

Lin, J., Handschin, C., Spiegelman, B. M. 2005. Metabolic control through the PGC-1 family of transcription coactivators. Cell Metabolism. 1 (6): 361-370. Doi: 10.1016/j.cmet.2005.05.004.

Mestroni, L. 2009, July 21. Phenotypic Heterogeneity of Sarcomeric Gene Mutations. A Matter of Gain and Loss?. Journal of the American College of Cardiology. 54 (4): 343-345. Doi: 10.1016/j.jacc.2009.04.029.

Motter, M. M., Corva, P., Krause, M., Perez Cenci, M., Soria, L. 2009. Rol de la calpastatina en la variabilidad de la terneza de la carne bovina. Journal of Basic and Applied Genetics. 20 (1): 15-24.

Norma Oficial Mexicana NOM-033-SAG/ZOO-2014, Métodos para dar muerte a los animales domésticos y silvestres. [Consultado 6 Junio 2019] 2014. Disponible en: https:// www.dof.gob.mx/nota_detalle.php?codigo $=5405210 \& \mathrm{fec}$ ha=26/08/2015.

Page, B. T., Casas, E., Heaton, M. P., Cullen, N. G., Hyndman, D. L., Morris, C. A., Crawford, A. M., Wheeler, T. L., Koohmaraie, M., Keele, J. W., Smith, T. P. L. 2002. Evaluation of singlenucleotide polymorphisms in CAPN1 for association with meat tenderness in cattle. Journal of Animal Science. 80 (12): 3077-3085. Doi: 10.2527/2002.80123077x.

Parra, B. G. M., Sifuentes, R. A. M., De la Rosa, R. X. F., Arellano, V.W. 2011. Avances y perspectivas de la biotecnología genómica aplicada a la ganadería en México. Tropical and Subtropical Agroecosystems. 14 (3): 1025-1037.

Rubio, L. M. de la S., Braña, V. D., Méndez, M. D., Torrescano U. G., Sánchez, E. A., Pérez, L. C., Figueroa, S. F., Delgado, S. E. 2013. Guía práctica para la estandarización y Evaluación de las Canales Bovinas mexicanas.1 ra. ed. Instituto Nacional de Investigaciones Forestales, Agrícolas y Pecuarias. México, D.F.

Sambrook, J., G. M. (2012). Molecular Cloning: A Laboratory Manual. 4 ed. Cold Spring Harbor Laboratory Press (Vol. 1).

Sifuentes, R. A.M., Puentes, M. H., Moreno, M. V.R., De la Rosa, R, X.F., Rosales, A, J. 2007. Frecuencia del alelo Q204X del gen Miostatina en hatos de Ganado del Noreste de México. Técnica Pecuaria en México. 45 (1): 85-92. Doi: 10.22319/ rmcp.v45i1.1785.

Sifuentes, R., María, A., Parra, B. M. G., De la Rosa, R. X. F., Sánchez, V. A., Serrano, M. F., Rosales, A. J. 2006. Importancia de las pruebas de paternidad basadas en microsatélites para la evaluación genética de ganado de carne en empadre múltiple. Técnica Pecuaria En México. 44 (3): 389-398.

Soria, L. A., Corva, P. M., Branda Sica, A., Villarreal, E. L., Melucci, L. M., Mezzadra, C. A., Papaleo, M. J., Fernadez, M. G., Silvestro, C., Schor, A., Miquel, M. C. 2009. Association of a novel polymorphism in the bovine PPARGC1A gene with growth, slaughter and meat quality traits in Brangus steers. Molecular and Cellular Probes. 23 (6): 304-308. Doi: 10.1016/j.mcp.2009.07.007.

Soria, L. A., Corva, P. M., Huguet, M. J., Miño, S., Miquel, M. C. 2010. Bovine $\mu$-calpain (CAPN1) gene polymorphisms in brangus and brahman bulls. BAG - Journal of Basic and Applied Genetics, 21 (1): 61-69. 
Valencia, D. C. E., y Zuluaga, J. J. E. 2013. Efecto del polimorfismo del gen de la somatotropina (BGH) sobre características lineales en vacas Holstein. Revista Lasallista de Investigación, 9(1), 21-31.

Van Eenennaam, A. L., Li, J., Thallman, R. M., Quaas, R. L., Dikeman, M. E., Gill, C. A., Franke, D.E., Thomas, M. G. 2007. Validation of commercial DNA tests for quantitative beef quality traits. Journal of Animal Science. 85 (4): 891-900. DOI: 10.2527/ jas.2006-512.
Zou, T., Mao, X., Yu, B., He, J., Zheng, P., Yu, J., Chen, D. 2014. Effects of dietary energy density and apparent ileal digestible lysine:digestible energy ratio on growth performance, meat quality, and peroxisome proliferator-activated receptor $\gamma$ (PPARY) gene expression of muscle and adipose tissues in LandracexRongchan. Livestock Science. 167 (1): 219-226. Doi: 10.1016/j.livsci.2014.04.032. 